\title{
INVENTÁRIO DE EMISSÕES DE GÁS DE EFEITO ESTUFA EM EMPRESA DE BASE FLORESTAL
}

\author{
GREENHOUSE GAS EMISSIONS INVENTORY \\ OF A FOREST-BASED COMPANY
}

scientific journal

v. 2 n. 22017

Recebido em 04/09/2017

Aceito em 05/11/2017

Jéssica Priscila Tosato ${ }^{1}$

Publicado em 06/11/2017

Allan Libanio Pelissari ${ }^{2}$

DOI: dx.doi.org/10.5380/biofix.v2i2.55069

Universidade Federal do Paraná, Curitiba, Paraná, Brasil tosatojessica@gmail.com ${ }^{1}$ \& allanpelissari@gmail.com ${ }^{2}$

\section{RESUMO}

A elaboração do inventário de emissões é o primeiro passo para que uma empresa contribua para a minimização das mudanças climáticas, uma vez que, a partir do diagnóstico obtido pelo inventário, a organização estabelece estratégias e metas para redução e para gestão das emissões de gases efeito estufa (GEE), empenhando-se na solução desse problema. Assim, o objetivo deste estudo foi obter o balanço entre as emissões e o sequestro de GEE pelos plantios de Eucalyptus sp. de uma empresa de base florestal. Para isso, quantificou-se o carbono emitido pelas operações florestais, desde a produção das mudas até o transporte dos toretes para a fábrica, bem como o carbono sequestrado pelos plantios florestais, e comparou-se com o ano base de 2015. A metodologia adotada para a quantificação das emissões baseiou-se nas especificações do IPCC. Considerando o balanço entre a captura e as emissões no sistema estudado conclui-se que $8,8 \%$ do $\mathrm{CO}_{2 \text { eq. }}$ removido foi efetivamente armazenado pelos plantios $(255.394,38 \mathrm{t})$, contribuindo positivamente para a redução do efeito estufa. Além disso, as emissões de $\mathrm{CO}_{2 \text { eq. }}$ reduziram em 2016 (20.036,08 t $\mathrm{CO}_{2 \text { eq. }}$ ), quando comparado ao ano base de 2015 (25.624,65 t CO 2 eq. $)$.

PALAVRAS-CHAVE: Balanço de emissões, Operações florestais, Sequestro de GEE.

\section{ABSTRACT}

Emission inventory developing is the first step for a company to contribute to minimizing climate changes, since the diagnosis obtained by inventory, the organization can be establishing strategies and targets for reduction and management of greenhouse gas emissions (GHG), working to solve this problem. Thus, the aim of this study was to obtain the balance between GHG emissions and sequestrations by Eucalyptus sp. of a forestbased company. Quantifying the carbon emitted by forestry operations from production of seedlings to transportation of logs to the factory. The methodology adopted for quantification of emissions is based on IPCC specifications. Considering the balance between emissions sequestrations in studied system, it is concluded that $8.8 \%$ of $\mathrm{CO}_{2 \text { eq }}$ removed is effectively stored by plantations $(255,394.38 \mathrm{t})$, contributing positively to reduction of greenhouse effect. In addition, $\mathrm{CO}_{2}$ eq emissions reduced in 2016 $\left(20,036.08 \mathrm{t} \mathrm{CO}_{2 \mathrm{eq}}\right)$, when compared the base year of 2015 $\left(25,624.65 \mathrm{t} \mathrm{CO}_{2 \mathrm{eq}}\right)$.

KEYWORDS: Emissions balance, Forest operations, GHG sequestration. 


\section{INTRODUÇÃO}

A interação entre a natureza e a ação humana, principalmente após a revolução industrial, foi marcada pela excessiva exploração dos recursos naturais, não considerando a frágil capacidade de regeneração e de assimilação do meio ambiente às mudanças abruptas. Devido a isso, surge a necessidade de um novo modelo de desenvolvimento consciente da sociedade que adote a sustentabilidade, visando desenvolver o presente de forma a deixar condições para que as gerações futuras também se desenvolvam (GEROMINI, 2004).

Há dois fatores notórios referentes as alterações de comportamento ambiental na superfície da Terra. O primeiro trata do aumento da temperatura média da superfície do planeta, enquanto o segundo fato está relacionado com o aumento da concentração de gases efeito estufa resultantes da combustão com carbono fóssil. Mesmo não existindo absoluto consenso da comunidade científica em relação a essas duas afirmações, não se pode deixar de notar que o planeta está sofrendo alterações devido as ações antrópicas. Isso implica em uma responsabilidade global na mitigação desses problemas (NAE, 2005; IBÁ, 2016).

Considerando esses aspectos, nas últimas décadas, surgiram debates mundiais a respeito de como a sociedade poderia minimizar os problemas das mudanças climáticas. Assim, alguns países iniciaram compromissos de diminuição de emissões de gases efeito estufa (GEE), bem como o fomento para iniciativas de novas tecnologias de baixo impacto ambiental e apoio às ideias de conservação da biodiversidade terrestre (NAE, 2005).

A participação com ações de mitigação não é realizada somente em âmbito global, podendo pequenas comunidades, instituições e empresas colaborarem para a minimização das mudanças climáticas. A execução de um inventário de emissões pode ser o primeiro passo que uma empresa pode tomar no apoio a solução desse problema. A realização de inventários de gases efeito estufa também permite às organizações visualizarem oportunidades de negócios no mercado de carbono, atrair novos investidores ou planejar processos que garantam a eficiência energética, operacional e econômica (GHG PROTOCOL, 2017).

Segundo o International Council of Forest and Paper Associations (ICFPA), a indústria global de base florestal é parte da solução para as mudanças climáticas. Com isso, esse setor tem alcançado progressos significativos na redução das emissões de carbono a partir de novas tecnologias, além do aumento do estoque de carbono e remoção de GEE, devido ao manejo sustentável das florestas (PAINEL FLORESTAL, 2016).

Nos últimos anos, é crescente o número de pesquisas que abordam esse tema na área florestal, devido as demandas por metodologias mais precisas para a quantificação do carbono. Em trabalhos como os de Gorgens et al. (2005), Soares et al. (2005) e Torres et al. (2013), busca-se comparar as melhores formas de quantificação da biomassa e de estocagem de carbono em florestas, a partir de modelos matemáticos e de ajustes metodológicos.

Geromini (2004) objetivou obter o balanço entre o carbono armazenado por plantios de Pinus taeda L. e o emitido pelas operações florestais e industriais. Como um dos resultados do balanço, foi observado que, se houver reposição do que foi colhido, haverá armazenamento de $\mathrm{CO}_{2}$ equivalente a $53,83 \%$ da quantidade de $\mathrm{CO}_{2}$ que entrou no processo industrial através da madeira em tora.

Segundo Higa et al. (2016), o Brasil possui elevado potencial para ações mitigadoras do efeito estufa. No entanto, é necessário que novos estudos sejam realizados para embasar uma nova gestão florestal, adequando-se aos efeitos das mudanças climáticas, permitindo o manejo sustentável das florestas, tanto em pequena escala como em grandes indústrias.

Muitos são os fatores que levam uma empresa de base florestal a realizar estudos do balanço de carbono de suas atividades. Entre eles, destacam-se os fatores políticos, econômicos, estratégicos e, em especial, as adequações de normas concomitantemente para o atendimento de exigências legais (GHG PROTOCOL, 2017). Destaca-se a ABNT NBR ISO 14.001/2015, que especifica os requisitos para um sistema de gestão ambiental, e a norma ABNT ISO/TR 14.069/2007, que estabelece orientações para os cálculos de emissões, a qual foi a principal motivação para a execução deste estudo.

O presente trabalho teve como objetivo obter o balanço entre as emissões e o sequestro de GEE pelos plantios de Eucalyptus sp. de uma empresa de base florestal. Para isso, quantificou-se o carbono emitido pelas operações florestais, desde a produção das mudas até o transporte dos toretes para a fábrica, e também o carbono sequestrado pelos plantios florestais, possibilitando comparar os resultados com o ano base de 2015.

\section{MATERIAL E MÉTODOS}

O projeto foi desenvolvido em uma empresa de base florestal que possui unidades no interior do estado de São 
Paulo, contemplando principalmente as regiões de Salto e Botucatu. As características de clima, solo e a localização das áreas estão especificadas na Tabela 1.

Tabela 1. Características de clima, solo e localização da empresa nas microrregiões de Botucatu e Salto

\begin{tabular}{ccc}
\hline Característica & \multicolumn{2}{c}{ Região } \\
\cline { 2 - 3 } & Botucatu & Salto \\
\hline Altitude (m) & 750 & 650 \\
Temperatura média anual & $19^{\circ} \mathrm{C}$ & $20^{\circ} \mathrm{C}$ \\
Temperatura mínima mensal & $15,2^{\circ} \mathrm{C} /$ julho & $15,9^{\circ} \mathrm{C} /$ julho \\
Temperatura máxima mensal & $21,9^{\circ} \mathrm{C} /$ fevereiro & $23^{\circ} \mathrm{C} /$ fevereiro \\
Clima Köppen & $\mathrm{Cfa}$ & $\mathrm{Cwa}$ \\
Solos predominantes & $\mathrm{LV}, \mathrm{LE}, \mathrm{RQ}$ & $\mathrm{LV}, \mathrm{PV}, \mathrm{Cb}$ \\
\hline
\end{tabular}

Cwa = Tropical Mesotérmico brando úmido, com 1 a 2 meses de seca; $\mathrm{Cfa}=$ Subtropical Mesotérmico brando úmido, com 1 a 2 meses de seca; $\mathrm{Cfb}=$ Temperado Mesotérmico brando semiúmido, sem seca. LV = Latossolo Vermelho amarelo; LE = Latossolo Vermelho escuro; PV = Podozólico Vermelho amarelo; $\mathrm{RQ}$ = Neossolo Quartzarênico; e $\mathrm{Cb}=$ Cambissolo.

\section{Quantificação das emissões de GEE}

A coleta de dados das emissões de GEE e os cálculos foram feitos de acordo com o IPCC (2006) no IPCC Guidelines for National Gas Inventories, revisado em 2010. As informações referentes a quantidade de combustível gasto no período de um ano foram disponibilizadas pelas empresas prestadoras de serviço das áreas de silvicultura e transporte. Nas atividades próprias de colheita e produção de mudas, as informações levantadas referem-se ao consumo de combustíveis, energia elétrica e resíduos gerados.

O estudo foi desenvolvido considerando os dados de emissões de GEE para o período de janeiro a dezembro de 2016, tendo o ano de 2015 como a base do inventário. Para a obtenção dos resultados do balanço de carbono entre as emissões e o sequestro de carbono, foram consideradas as informações referentes somente as atividades florestais, desde a obtenção das mudas de Eucalyptus sp. até a entrega dos toretes na fábrica de painéis e pisos de madeira.

Neste estudo, foram consideradas as várias idades do plantio de Eucalyptus sp. e as principais operações responsáveis pela emissão de $\mathrm{CO}_{2}$, incorporando as emissões por recursos não renováveis, contemplando as seguintes operações:

I. Uso de veículos próprios para transporte de funcionários;

II. Veículos alugados para transporte de funcionários;
III. Máquinas florestais para a silvicultura;

IV. Máquinas florestais para colheita e arraste;

V. Uso de motosserra; e

VI. Composição veicular para transporte de toras/toretes.

Outras emissões de dióxido de carbono também foram consideradas, advindas de energia elétrica, resíduos orgânicos (alimentos) e resíduos da colheita. $\mathrm{Na}$ Tabela 2 estão descritas as informações coletadas por atividade.

Tabela 2. Dados coletados em uma empresa florestal para o cálculo de emissões de $\mathrm{CO}_{2}$ por atividade no período de um ano

\begin{tabular}{c|l}
\hline Atividade & \multicolumn{1}{c}{ Consumo } \\
\hline $\begin{array}{c}\text { Viveiro de } \\
\text { produção de mudas }\end{array}$ & $\begin{array}{l}\text { - Energia Elétrica (Kwh/mês) } \\
\text { Combustível gasto no transporte de funcionários } \\
\text { (L/mês ou L/ano) }\end{array}$ \\
\hline Combustíveis de máquinas e tratores (L/mês ou \\
L/ano)
\end{tabular}

Para a obtenção dos valores de $\mathrm{CO}_{2}$ emitidos pelos processos florestais, foi considerado o tipo e a quantidade anual de combustível utilizado em cada fonte, multiplicando-se por um fator, variando conforme o tipo de dado coletado (combustível ou energia elétrica gastos). Esses fatores foram adaptados de uma listagem fornecida pelo IPCC (2010), que permite obter o valor direto de $\mathrm{CO}_{\text {2eq. }}$ (1).

$E_{\text {eq. }}=V \cdot f$

Em que: $E_{\text {eq. }}=$ tonelada equivalente de $\mathrm{CO}_{2}$; $V=$ quantidade de combustível (L) ou energia (Kwh); e $f=$ fator de conversão, sendo 0,003 para combustível e 0,04 para energia. 
Para o cálculo de emissões de $\mathrm{CO}_{2 \text { eq. }}$ provenientes dos resíduos orgânicos e da colheita, foi necessário primeiramente determinar a quantidade de metano $\left(\mathrm{CH}_{4}\right)$ produzido, utilizando para isso um fator de conversão para metano (2). Posteriormente, efetuou-se o cálculo da emissão de $\mathrm{CO}_{2 \text { eq. }}$ multiplicando o volume de metano pelo fator de conversão do $\mathrm{CO}_{2 \text { eq. }}$ (3).

$E_{C H 4}=V \cdot f_{C H 4}$

$E_{\text {eq. }}=V_{C H 4} * f$

Em que: $E_{C H 4}=$ toneladas equivalentes em metano; $V$ = quantidade de resíduo $(\mathrm{t}) ; V_{C H 4}=$ volume de metano; $\mathrm{f}_{\mathrm{CH} 4}=$ fator de conversão para metano, sendo 0,5 para resíduo florestal e 0,15 para resíduo alimentar; e $f=$ fator de conversão correspondente a 0,318 para resíduos.

\section{Balanço de carbono}

Em 2014, o setor de pesquisa e desenvolvimento da empresa realizou o inventário da biomassa. Na estimativa da biomassa total das árvores, foi considerada a relação entre as partes dos componentes das árvores e a biomassa do tronco, obtida pelo método destrutivo, por apresentar bom grau de confiabilidade dos valores gerados em relação à biomassa real (NEVES, 2000; GATTO et al., 2011), sendo posteriormente aplicadas equações de regressão.

A biomassa dos componentes das árvores foi convertida em estoque de carbono (C), multiplicando a biomassa encontrada pelo teor de $C$ igual a 0,5 por meio da seguinte fórmula (4) (IPCC, 2006).

$t C O_{2 e q .}=\operatorname{vol} \cdot C \cdot \frac{44}{12}$

Em que: $t \mathrm{CO}_{2 \text { eq. }}=$ dióxido de carbono equivalente, em toneladas; $v o l=$ Volume de biomassa $(\mathrm{t})$ dos componentes das árvores; e $C=$ teor de carbono constante, igual a 0,5.

A partir do resultado do inventário de biomassa e carbono realizado na empresa, foi efetuada a comparação entre a quantidade de GEE emitido e o incremento médio anual de carbono dos plantios. Obteve-se o balanço geral de emissões a partir da diferença entre a taxa anual de fixação de carbono pelo sumidouro (plantio) e as emissões totais de $\mathrm{CO}_{2 \text { eq. }}$ produzidas no período.

O saldo de balanço de carbono foi obtido pela diferença entre a remoção de $\mathrm{CO}_{2 \text { eq }}$ pelos plantios e a emissão. A equação (5) apresenta o cálculo para o saldo.

$$
\text { Saldo }=\left(\text { Remoç2015 }_{20}-\text { Remoç2016 }_{20}\right)+\left(\text { Emiss }_{2016}\right)
$$

Em que: Remoç = remoção de $\mathrm{CO}_{2 \text { eq.; }}$ e Emiss = emissão de $\mathrm{CO}_{2 \text { eq. }}$.

\section{RESULTADOS E DISCUSSÃO}

\section{Fixação do carbono pelos plantios}

A análise comparativa da quantidade de carbono removidos pelos plantios de Eucalyptus sp. nos anos de 2015 e 2016 revelou que o total de $\mathrm{CO}_{2 \text { eq. }}$ sequestrado em 2016 superou em 8,76\% (255.394,22 t CO 2eq.) as remoções do ano base (2015). Nas Tabelas 3 e 4 estão descritos os valores obtidos de biomassa por classes de idade, bem como a quantidade de $\mathrm{CO}_{2 \text { eq. }}$ removido no período de estudo.

Observando a porcentagem de dióxido de carbono que os plantios removeram nas diferentes classes de idades, a maior remoção no ano de 2015 foi nos plantios entre 4 e 5 anos (31\%), superando o ano de 2016 (21\%). A maior porcentagem de $\mathrm{CO}_{2 \text { eq. }}$ removida foi nos plantios entre 5 e 6 anos em 2016.

$\mathrm{Na}$ literatura, diversos trabalhos com diferentes espécies florestais apontam para um maior aumento na quantidade de carbono estocado na biomassa com o aumento da idade da floresta, até um certo limite (SANQUETTA, 2002; GEROMINI, 2004; WATZLAWICK, 2005). As florestas mais antigas dentem ao equilíbrio, reduzindo a fixação do carbono com o passar dos anos.

Muito embora, os resultados observados na fixação do carbono em diferentes classes de idade sejam relevantes, é necessária cautela na comparação dos dados nos diferentes anos, visto que a área do plantio mudou de um ano para outro, ocorrendo o acréscimo de 590,47 ha em 2016. Outros fatores que afetam a produção de biomassa, e, consequentemente, influenciam nos resultados das remoções de $\mathrm{CO}_{2 \text { eq. }}$ sendo: capacidade genética da espécie, idade, sítio e densidade do povoamento. Todos esses fatores, quando possível, devem ser analisados separadamente para um melhor controle dos resultados.

\section{Emissões de carbono}

O estudo aponta que as emissões reduziram em $21,8 \%$ no ano de 2016 (Tabela 5). O valor total das emissões em 2015 foi de 25.624,65 t de $\mathrm{CO}_{2 \text { eq., }}$ ao passo que houve redução para 20.036,08 t de $\mathrm{CO}_{2 \text { eq. }}$ em 2016. Em ambos os períodos, o setor de transporte de toretes foi o responsável pelos maiores níveis de emissões, seguido da colheita e da silvicultura. 
Tabela 3. Quantidade de $\mathrm{CO}_{2 \text { eq. }}$ removidos da atmosfera no ano de 2015 e volume de biomassa inventariados por classe de idade em uma empresa de base florestal

\begin{tabular}{|c|c|c|c|c|c|c|c|c|c|c|}
\hline \multirow[b]{2}{*}{ Idade } & \multirow{2}{*}{$\begin{array}{l}\text { Área total } \\
\text { (ha) }\end{array}$} & \multicolumn{7}{|c|}{ Biomassa (t) } & \multicolumn{2}{|c|}{$\mathrm{CO}_{\text {eq. }}$ removidos } \\
\hline & & Casca & Casca & Galhos & Lenho & Raízes & Total & t/ha & t/ha & $\%$ \\
\hline 2 a 2,9 & $4.929,44$ & $16.211,26$ & $17.162,01$ & $17.664,69$ & $146.273,89$ & $30.693,61$ & $228.005,47$ & 46,25 & $418.010,02$ & 16 \\
\hline 3 a 3,9 & $3.980,22$ & $18.963,70$ & $9.296,47$ & $13.876,48$ & $187.289,38$ & $28.435,76$ & $257.861,80$ & 64,79 & $472.746,64$ & 18 \\
\hline 4 a 4,9 & $5.392,91$ & $32.113,11$ & $15.121,92$ & $18.472,81$ & $339.382,82$ & $42.694,29$ & $447.784,96$ & 83,03 & $820.939,09$ & 31 \\
\hline 5 a 5,9 & $3.011,27$ & $21.561,77$ & $8.394,29$ & $10.898,46$ & $261.818,43$ & $27.878,12$ & $330.551,07$ & 109,77 & $606.010,29$ & 23 \\
\hline 6 a 6,9 & 783,21 & $6.273,67$ & $2.328,42$ & $3.178,37$ & $95.298,67$ & $8.837,88$ & $115.917,00$ & 148,00 & $212.514,50$ & 8 \\
\hline 7 a 7,5 & 376,31 & $2.884,31$ & $1.187,30$ & $1.680,81$ & $59.369,47$ & $4.965,91$ & $70.087,79$ & 186,25 & $128.494,28$ & 5 \\
\hline Total & $18.473,36$ & & & & & & & & $2.658 .714,83$ & 100 \\
\hline
\end{tabular}

Tabela 4. Quantidade de $\mathrm{CO}_{2 \text { eq. }}$ removidos da atmosfera no ano de 2016 e volume de biomassa inventariados por classe de idade em uma empresa de base florestal

\begin{tabular}{|c|c|c|c|c|c|c|c|c|c|c|}
\hline \multirow{2}{*}{ Idade } & \multirow{2}{*}{$\begin{array}{l}\text { Área total } \\
\text { (ha) }\end{array}$} & \multicolumn{7}{|c|}{ Biomassa (t) } & \multicolumn{2}{|c|}{$\mathrm{CO}_{\text {2eq. }}$ removidos } \\
\hline & & Casca & Casca & Galhos & Lenho & Raízes & Total & t/ha & t/ha & $\%$ \\
\hline 2 a 2,9 & $3.746,32$ & $13.062,32$ & $12.846,42$ & $13.411,00$ & $119.168,66$ & $23.857,89$ & $182.346,29$ & 48,67 & $334.301,54$ & 11 \\
\hline 3 a 3,9 & $4.872,76$ & $23.295,16$ & $11.169,80$ & $16.982,14$ & $230.441,77$ & $34.858,44$ & $316.747,31$ & 65,00 & $580.703,39$ & 20 \\
\hline 4 a 4,9 & $3.914,80$ & $23.437,41$ & $10.963,02$ & $13.415,85$ & $248.395,66$ & $31.097,41$ & $327.309,34$ & 83,61 & $600.067,12$ & 21 \\
\hline 5 a 5,9 & $5.228,85$ & $37.207,39$ & $14.548,58$ & $18.852,20$ & $449.058,99$ & $48.068,67$ & $567.735,83$ & 108,58 & $1.040 .849,02$ & 36 \\
\hline 6 a 6,9 & $1.131,35$ & $9.047,81$ & $3.354,73$ & $4.572,39$ & $136.174,40$ & $12.682,12$ & $165.831,45$ & 146,58 & $304.024,33$ & 10 \\
\hline 7 a 7,5 & 169,75 & $1.351,96$ & 527,96 & 738,92 & $24.783,01$ & $2.141,97$ & $29.543,81$ & 174,04 & $54.163,65$ & 2 \\
\hline Total & $19.063,83$ & & & & & & & & $2.914 .109,05$ & 100 \\
\hline
\end{tabular}

Tabela 5. Emissões de $\mathrm{CO}_{2 \text { eq. }}$ por setor produtivo de uma empresa de base florestal, correspondente aos anos de 2015 e 2016

\begin{tabular}{ccccc}
\hline \multirow{2}{*}{ Setor } & \multicolumn{2}{c}{$\mathbf{C O}_{\text {2eq. }}$ emitidos } & \multicolumn{2}{c}{$\% \mathrm{CO}_{\text {2eq. }}$} \\
\cline { 2 - 5 } & $\mathbf{2 0 1 5}$ & $\mathbf{2 0 1 6}$ & $\mathbf{2 0 1 5}$ & $\mathbf{2 0 1 6}$ \\
\hline $\begin{array}{c}\text { Viveiro de } \\
\text { produção de mudas }\end{array}$ & 18,51 & 35,55 & 0,1 & 0,18 \\
Silvicultura & 772,92 & $1.225,55$ & 3,0 & 6,12 \\
\hline Colheita & $6.311,87$ & $5.630,76$ & 24,6 & 28,10 \\
\hline Transporte de toretes & $18.053,38$ & $12.790,34$ & 70,5 & 63,84 \\
\hline Administrativo & 391,00 & 252,51 & 1,5 & 1,26 \\
\hline Almoxarifado & 76,99 & 101,37 & 0,3 & 0,51 \\
\hline Total & $25.624,65$ & $20.036,08$ & 100 & 100 \\
\hline
\end{tabular}

Esse resultado está de acordo com a média nacional de emissões, que aponta o setor de transportes como o maior responsável pelas emissões, seguido pelo setor industrial (BILLER e GOLDEMBERG, 1999). Geromini (2004) também relata que, ao analisar as atividades florestais na cadeia produtiva de molduras, o setor de transporte é responsável pelos maiores valores de emissões de $\mathrm{CO}_{2 \text { eq. }}$.

Foi observado sutil aumento das emissões no viveiro de produção de mudas do ano de 2015 para 2016. Esse resultado pode ser explicado, dentre outros fatores, pelo acréscimo da área plantada no período, o que resultou em maior demanda por mudas e, consequentemente, influenciou no aumento das emissões. A silvicultura foi outro setor que intensificou suas emissões, em razão do maior gasto com combustível das máquinas florestais para as atividades de preparo de solo e plantio.

Em função da crise econômica, a empresa tomou medidas de redução de custos, reduzindo os gastos com combustíveis e impactando na redução das emissões. Além dessa medida, outro fator que justifica a redução das emissões em 2016 foi a menor demanda de madeira pela indústria, diminuindo a quantidade de material colhido e consequentemente transportado para as unidades fabris.

$\mathrm{Na}$ Figura 1 estão indicadas as porcentagens de emissões de $\mathrm{CO}_{2 \text { eq. }}$ para os anos de 2015 (Figura 1A) e 2016 (Figura 1B), respectivamente. 
(A) Emissões 2015

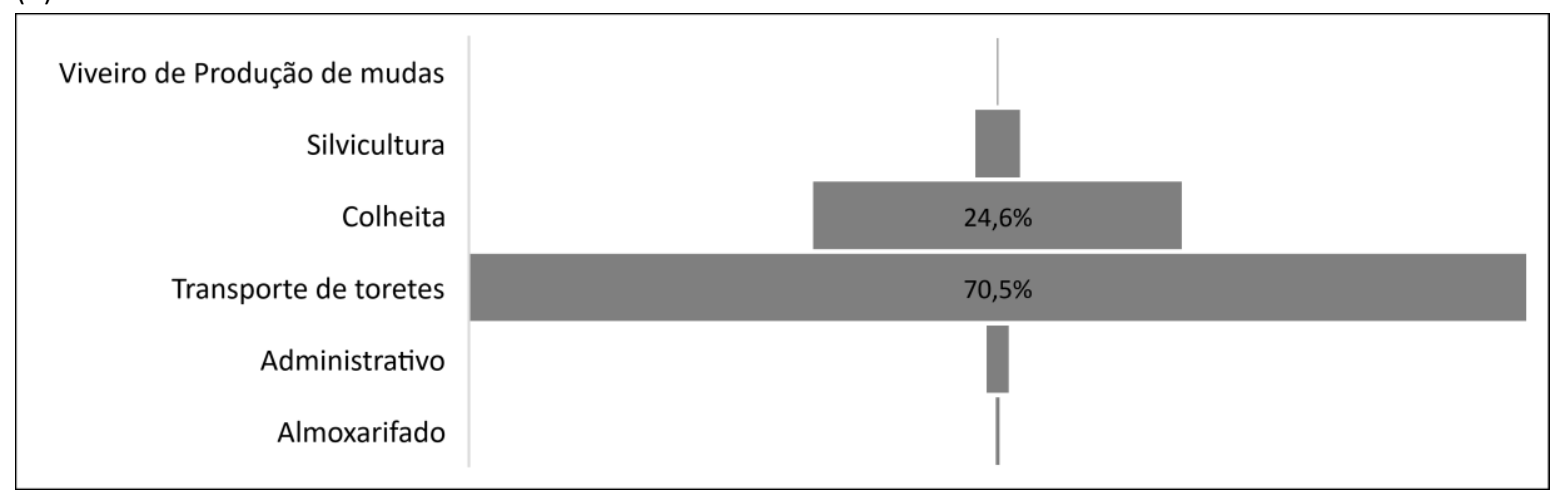

(B) Emissões 2016

\begin{tabular}{|r|c|}
\hline Viveiro de Produção de mudas & \\
Silvicultura & $6,2 \%$ \\
Colheita & $28,0 \%$ \\
Transporte de toretes & $64,0 \%$ \\
Administrativo & \\
Almoxarifado & \\
\hline
\end{tabular}

Figura 1. Porcentagem de emissões de $\mathrm{CO}_{2 \text { eq. }}$ calculados para os anos 2015 e 2016 referente as atividades de uma empresa de base florestal.

\section{Balanço de carbono}

A análise do balanço final entre as remoções e as emissões da empresa em 2016 revelou que as remoções superaram as emissões em 275.430,5 t de $\mathrm{CO}_{2 \text { eq. }} \mathrm{O}$ saldo foi positivo, uma vez que a remoção efetiva no ano de 2016, sendo o volume removido de 2015 subtraído da quantidade removida em 2016, foi de 255.394,4 t de $\mathrm{CO}_{2 \text { eq. }}$. No ano base, o saldo apresenta-se negativo, pois o

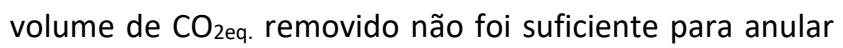
as emissões de 2015 (Tabela 6).

Tabela 6. Balanço de carbono calculado para os anos de 2015 e 2016, referente as atividades de uma empresa de base florestal

\begin{tabular}{cccc}
\hline & Unidade & $\mathbf{2 0 1 5}$ & $\mathbf{2 0 1 6}$ \\
\hline Biomassa & $\mathrm{t}$ & $1.450 .208,00$ & $1.589 .514,03$ \\
Remoção & $\mathrm{t} \mathrm{CO}_{\text {2eq. }}$ & $2.658 .714,67$ & $2.914 .109,05$ \\
Remoção efetiva no ano & $\mathrm{t} \mathrm{CO}_{\text {2eq. }}$ & $-190.075,54$ & $255.394,38$ \\
\hline Emissão & $\mathrm{t} \mathrm{CO}_{\text {2eq. }}$ & $25.624,65$ & $20.036,08$ \\
Saldo & $\mathrm{t} \mathrm{CO}_{\text {2eq. }}$ & $-164.450,89$ & $275.430,47$ \\
\hline
\end{tabular}

\section{CONCLUSÕES}

Considerando o balanço entre a captura e as emissões no sistema estudado, $8,8 \%$ do $\mathrm{CO}_{2 \text { eq. }}$ removido é efetivamente armazenado pelos plantios (255.394,38 t), contribuindo positivamente para a redução do efeito estufa. As emissões de $\mathrm{CO}_{\text {2eq. }}$ reduziram em 2016 $\left(20.036,08\right.$ t $\left.\mathrm{CO}_{2 \text { eq. }}\right)$ quando comparadas ao ano base de $2015(25.624,65$ t CO 2eq.).

\section{REFERÊNCIAS}

ABNT - ASSOCIAÇÃO BRASILEIRA DE NORMAS TÉCNICAS. ABNT ISO/TR 14.064 - Gases de efeito estufa. Rio de Janeiro, 2007.

ABNT - ASSOCIAÇÃO BRASILEIRA DE NORMAS TÉCNICAS. ABNT NBR ISO 14.001 - Sistema de Gestão Ambiental. Rio de Janeiro, 2015.

BILLER, D.; GOLDEMBERG, J. Efeito estufa e a convenção sobre as mudanças climáticas. Ministério da Ciência e Tecnologia, Brasília, 1999.

GATTO, A.; BARROS, N. F.; NOVAIS, R. F.; SILVA, I. R.; LEITE, H. G.; VILLANI, E. M. A. Estoque de carbono na biomassa de plantações de eucalipto na região Centro-Leste do estado de Minas Gerais. Revista Árvore, v. 35, n. 4, p. 895-905, 2011.

GEROMINI, M. P. Análise qualitativa do balanço de carbono em empresa do setor florestal destinada à produção de molduras. 2004. 117 f. Dissertação (Mestrado em Engenharia Ambiental) Universidade Regional de Blumenau, Blumenau, 2004.

GHG PROTOCOL. Contabilização, quantificação e publicação de inventários corporativos de emissões de gases de efeito estufa. 2. ed., 2017. Disponível em: <http://ghgprotocolbrasil.com.br/ 
especificacoes-e-notas-tecnicas-do-programa-brasileiro-ghgprotocol/?locale=pt-br>. Acesso em: 02/05/2017.

GORGENS, E. R.; OLIVEIRA, M. L. R.; LEITE, H. G.; BRUM NETO, M. S. Metodologia para monitoramento do estoque de carbono: revisão e estudo de caso. Biomassa \& Energia, v. 2, n. 2, p. 169175, 2005.

HIGA, R. C. V.; ZANATTA, J. A.; RACHWALL, M. F. Dinâmica da emissão de gases de efeito estufa e dos estoques de carbono em florestas naturais e plantadas. In: Jornadas Técnicas Forestales y Ambientales, 17., Misiones, Anais..., Misiones, p. 17-19, 2016. Disponível em: <https://ainfo.cnptia.embrapa.br/digital/ bitstream/item/148754/1/2016-RosanaH-JOTEFA-

Dinamica.pdf>. Acesso em: 01/04/2017.

IBÁ - Indústria Brasileira de Árvores. Relatório anual 2016. 2016. 100 p. Disponível em: <http://iba.org/pt/sala-deimprensa/releases/9-conteudo-pt/715-iba-publica-relatorioanual-2016> Acesso em: 01/04/2017.

IPCC - Intergovernmenttal Panel on Climate Change. 2006 IPCC Guidelines for National Greenhouse Gas Inventories. 2006. Disponível em: <http://www.ipcc-nggip.iges.or.jp/public/ 2006gl> Acesso em: 02/05/2017.

IPCC - Intergovernmenttal Panel on Climate Change. 2006 IPCC Guidelines for National Greenhouse Gas Inventories. volume 2. energy, corrected chapter in 2010. 2010. Disponível em: <http://www.ipcc-nggip.iges.or.jp/public/2006gl> Acesso em: 02/03/2017.

NAE - Núcleo de Assuntos Estratégicos da Presidência da República. Mudanças Climáticas: Negociações internacionais sobre a mudança do clima. Brasília, 2005. 252 p. Disponível em: <http://livroaberto.ibict.br/bitstream/1/574/2/caderno mudan casclimatisimpactos.pdf $>$. Acesso em: 22/04/2017.

NEVES, J. C. L. Produção e partição de biomassa, aspectos nutricionais e hídricos em plantas clonais de eucalipto na região litorânea do Espirito Santo. 2000. 202 f. Tese (Doutorado em Produção Vegetal) - Universidade Estadual do Norte Fluminense, Rio de Janeiro, 2000.

PAINEL FLORESTAL. Indústria global de base florestal é parte da solução para mudanças climáticas. 2016. Disponível em: <http://www.painelflorestal.com.br/noticias $>$. Acesso em: $16 / 03 / 2017$.

SANQUETTA, C. R.; WATZALAWICH, L. F.; BALBINOT, R. M.; ZILIOTTO, M. A. B.; GOMES, F. dos S. As florestas e o carbono. Curitiba: FUPEF/UFPR, 2002. 264 p.

SOARES, C. P. B.; LEITE, H. G.; GÖRGENS, E. B. Equações para estimar o estoque de carbono no fuste de árvores individuais e em plantios comerciais de eucalipto. Revista Árvore, v. 29, n. 5, p. 711-718, 2005.

TORRES, C. M. M. E.; JACONINE, L. A. G.; SOARES, C. P.; OLIVEIRA NETO, S. N.; SANTOS, R. D.; CASTRO NETO, F. Quantificação de biomassa e estocagem de carbono em uma floresta estacional semidecidual, no Parque Tecnológico de Viçosa, MG. Revista Árvore, v. 37, n. 4, p. 647-655, 2013.
WATZLAWICK, L. F.; SANQUETTA, C. R.; CALDEIRA, M. V. W. Estoque de carbono orgânico e biomassa em Pinus taeda $\mathrm{L}$. Renabio, v. 2, n. 1, p. 7-17, 2005. 